\title{
Inspiratory and Expiratory Resistances During Exercise
}

\author{
Arthur T. Johnson ${ }^{1 *}$, Prakash Chapain ${ }^{1}$, Darnell Slaughter ${ }^{1}$, \\ Sally Gallena ${ }^{2}$ and Jafar Vossoughi ${ }^{3}$ \\ ${ }^{1}$ Fischell Department of Bioengineering, University of Maryland, College Park, MD 20742, \\ USA. \\ ${ }^{2}$ Loyola University Maryland, 8890 McGaw Rd., Columbia, MD 21045, USA. \\ ${ }^{3}$ Engineering and Scientific Research Associates, Olney, MD 20832, USA.
}

\section{Authors' contributions}

This work was carried out in collaboration between all authors. Author ATJ conceived of and planned the study, authors PC and DS performed the tests, author SG provided medical expertise for paradoxical vocal fold motion, and author JV gave significant general advice and helped provide funding. All authors read and approved the final manuscript.

Research Article

Received $26^{\text {th }}$ October 2012 Accepted $19^{\text {th }}$ December 2012 Published $2^{\text {nd }}$ April 2013

\section{ABSTRACT}

Aims: Paradoxical vocal fold motion, especially during exercise, causes symptoms of dyspnea in patients experiencing this condition. At present, the standard means to diagnose this condition is invasive using a laryngoscope. The Airflow Perturbation Device (APD) could offer a simpler means of diagnosis and monitoring, but the APD must be validated with laryngoscopy. Both devices require access to the mouth, and so cannot be used simultaneously. The aim of this study was to determine if respiratory resistance of exercising subjects changes immediately after exercise begins and ends.

Study Design: The study was conducted as a prospective study.

Place and Duration of Study: All tests were conducted in the Human Performance Laboratory, Fischell Department of Bioengineering, University of Maryland, College Park, MD between August 2011 and August 2012.

Methodology: Fifteen subjects exercised on a bicycle ergometer at $70 \%$ of maximum predicted heart rate while breathing through the APD.

Results: Results show that APD measurements made just prior and after the cessation of

*Corresponding author: Email: artjohns@umd.edu; 
exercise are comparable.

Conclusion: APD measured inspiration and expiration resistances do not change immediately after exercise cessation.

Keywords: Airflow perturbation device; exercise flow rates; respiratory resistance; vocal cord dysfunction.

\section{INTRODUCTION}

Paradoxical Vocal Fold Motion (PVFM), previously known as Vocal Cord Dysfunction (VCD), is a condition where the vocal folds adduct (close) during inspiration when they should abduct (open), thus decreasing airway patency. Although sporadic case reports have described symptoms suggestive of PVFM in the early literature, the disorder had not gained recognition until the past 15 years. Patients experience breathing difficulty, usually triggered by exercise, and seek medical amelioration [1-2]. They are commonly misdiagnosed as asthmatics, yet they do not respond to asthma therapy. Accurate and timely diagnoses are needed before satisfactory treatments can be developed, but there are presently no standardized methodologies for PVFM assessment [3].

At present, the standard assessment procedure uses a laryngoscope to visualize the vocal cords [4], although other pulmonary function tests have also been used [5-8]. Adduction of the vocal folds is mostly seen during inspiration, but it can continue into expiration as well [9]. However, adduction during expiration alone should not be considered to represent PVFM, because this can be caused by an adaptation to lower airway constriction [9].

Laryngoscopy has not been found to be well tolerated by exercising patients in our clinic. The optical fiber laryngoscope must be positioned deep in the oropharynx for proper visualization, and gagging is common. Although there may be other triggers for the condition, exercise frequently brings on exercise-induced PVFM (EIPVFM) for those susceptible. EIPVFM may subside soon after exercise ceases, so laryngoscopy must be performed within a very short time after exercise ceases. Whereas other well-trained professionals may be able to administer laryngoscopic measurements during the exercise stage of testing, others, including ourselves, have found it to be nearly impossible to perform during exercise. Thus, we have searched for an alternative means to indicate when EIPVM is likely.

The Airflow Perturbation Device (APD) is an experimental instrument to measure respiratory resistance. It consists of a segmented wheel rotating in the breathing flow pathway [10]. Part of the wheel is open, and part screened, so that, as it rotates, it causes flow to periodically diminish and grow. Measured mouth pressure is also perturbed. The depth of each of these perturbations depends upon two things: resistance of the device and respiratory resistance of the patient. Measuring both mouth pressure and flow easily gives the resistance of the device, so patient respiratory resistance can be obtained noninvasively. Values obtained with the APD are comparable to those measured by impulse oscillometry (IOS), a form of forced oscillation (FO) [11].

The APD is relatively small and easy to use. It requires only about a minute of normal breathing to make its measurement. Of particular interest to PVFM diagnosis, it can separate 
respiratory resistance during inspiration from that during expiration. Thus, the APD could become a very important noninvasive tool to diagnose PVFM.

However, in order to ascertain the utility of the APD in EIPVFM diagnosis, APD measurements must be compared directly with laryngoscope findings. That produces a physical conflict, because the APD requires a seal with the mouth or nose to measure properly [12], yet the laryngoscope requires access to the throat. One possible means around this conundrum is to measure inspiratory and expiratory resistances immediately before exercise ceases with the APD, and immediately after exercise cessation with the laryngoscope. This procedure could be justified if the APD measured the same resistances immediately before and after exercise stopped. It is the purpose of this study to determine if resistances during inspiration and expiration change or remain the same on either side of the exercise transition. If there is no change, then laryngoscope and APD diagnoses could be directly compared. In addition, measurements of respiratory resistance before, during, and immediately after exercise could be of interest to pulmonologists interested in respiratory mechanics.

\section{MATERIALS AND METHODS}

Fifteen healthy non-asthmatic male and female subjects aged 18 years or older volunteered for respiratory resistance measurements at rest, during exercise, and at post-exercise rest. All subjects were students at the University of Maryland College Park and the protocol for human testing was approved by the University of Maryland Institutional Review Board. Before each test session, each subject completed a brief Medical History Questionnaire and a Physical Readiness Questionnaire for subject screening purposes. Only subjects presumed fit for exercise were allowed to participate in the test session. All subjects exercised by pedaling on a bicycle ergometer (Life Cycle 9500HR) and respiratory resistance measurements were taken in a seated position while breathing through the APD before, during, and after exercise. They breathed through the mouth only, a nose clip obstructing nasal flow.

The APD used in this study was calibrated for resistance values by comparison with a commercially-available Impulse Oscillometry (IOS) system (Care Fusion Master Screen; San Diego, CA) using a physical mechanical analog of the respiratory system [13]. Because the IOS cannot distinguish between resistances during inspiration from those during expiration, the APD was then calibrated with a three liter syringe (flow) and manometer (pressure) for resistances in both inspiration and expiration directions following the procedure in [10].

\subsection{Initial Exercise Calibration}

Each subject was tested on the bicycle ergometer prior to the data collection to determine the proper settings to elicit $70 \%$ of the subject's predicted maximum heart rate. Subjects were seated on the ergometer which measures the amount of work done and asked to pedal at a rate of 80-90 rpm. The heart rate was monitored using the sensors on the ergometer. The ergometer settings were adjusted until the heart rate reached a steady state value of about $70 \%$ of age-predicted maximum heart rate. 


\subsection{Experimental Session and Respiratory Resistance Measurements}

Following the exercise calibration, subjects were allowed to rest for a long enough time for the heart rate to return to normal resting values. The subjects were then asked to sit on the ergometer and rest while respiratory resistance measurements were continuously acquired and recorded for a minute using the APD and the APD computer program. Subjects were then asked to begin pedaling the ergometer using the predetermined settings that would elicit a heart rate of $70 \%$ of age-predicted maximum heart rate. Each subject's heart rate was monitored using the sensors on the ergometer. Subjects exercised on the ergometer for five minutes while breathing through the APD. Pedaling ceased at five minutes, and the post-exercise rest period continued for two and a half additional minutes. The total time for data collection was eight and one half minutes.

\subsection{Data Analysis}

Individual APD readings exhibit a moderate amount of variability, and this variability can obscure recognition of real resistance changes. At least some of this variability appears to come from dynamic resistance changes of physiological origin in the respiratory system [13]. The APD, when used as a stand-alone device, averages individual readings for about one minute to produce a reasonably stable resistance reading. When used with a computer, however, individual APD resistance readings are accessible, and these were used for this study. To dampen variation of these readings, each reading was averaged over readings obtained for ten seconds, and labeled with the time corresponding to the average time of the ten second sample. Samples overlapped by nine seconds so that an averaged reading was available for each second from time beginning at 5 seconds to time ending at 510 seconds. The number of samples for each of these time intervals varied, because resistance was calculated once for each airflow perturbation, but wheel rotation speed can vary somewhat, especially during the intense breathing of exercise. APD pressure and flow data were collected at $200 / \mathrm{sec}$, but times for perturbations usually slowed during inspiration, when the wheel was pulled closer to the APD body, and became faster during expiration.

Data for the entire cohort of participants were analyzed using the Student's t-test [14]. This test was performed for six conditions: difference of APD inspiratory resistance $\left(R_{i}\right)$ immediately before and resistance immediately after exercise ceased (two readings total, denoted as local differences), and the same for expiratory resistances $\left(R_{e}\right)$; differences of $R_{i}$ averaged for 30 seconds immediately before and 30 seconds immediately after exercise ceased (60 readings total), and the same for $R_{e}$; and differences of $R_{i}$ averaged for 60 seconds immediately before and 60 seconds immediately after exercise ceased (120 readings total), and the same for $R_{\mathrm{e}}$. These values of Student's $t$ were compared to twotailed (sign ignored) tabled values at the $p=0.05$ level with 14 degrees of freedom [14]. Data for the entire cohort of participants were also analyzed using a t-test with paired means using resistance differences before and after exercise ceased.

In order to investigate whether each individual subject's $R_{i}$ and $R_{e}$ immediately before exercise ceased could be considered to be the same as $R_{i}$ and $R_{e}$ immediately after, the same ratios as described above for the entire cohort were calculated for each subject. Ratios of sample averages divided by appropriate standard deviations were calculated for three inspiratory and three expiratory conditions: $R_{i}$ and $R_{e}$ immediately on each side of exercise cessation (local), $R_{i}$ and $R_{e}$ averaged for 30 seconds before and after, and $R_{i}$ and $R_{e}$ averaged 60 seconds before and after. Lacking a standard deviation for one local reading, 
the standard deviation for the entire eight and one-half minute test was used as the denominator for the local ratios, but standard deviations for the 30 second and 60 second ratios used standard deviations calculated for each of these time intervals. It was not appropriate to use a t-test on the local ratios because of the use of the entire sample standard deviation, but the 30 second and 60 second ratios were tested at the $p=0.05$ level with 58 and 118 degrees of freedom, respectively.

All calculations were performed using the Microsoft (Redman, WA) Excel program.

\section{RESULTS AND DISCUSSION}

In Table 1 are shown demographic characteristics for the 15 subjects. In general, they were all young, healthy adult college students.

Table 1. Subject demographics. Values given are means \pm standard deviation and ranges

\begin{tabular}{ll}
\hline Sex & 11 male, 4 female \\
Age (years) & $22.3 \pm 3.4 \quad(19$ to 30$)$ \\
Height $(\mathrm{cm},[\mathrm{in}])$ & $173 \pm 6.9 \mathrm{~cm}[68.1 \pm 2.7 \mathrm{in}] 165$ to $185 \quad$ [65 to 73$]$ \\
Body mass $(\mathrm{kg},[\mathrm{lb}])$ & $68.2 \pm 8.9 \mathrm{~kg}[150.1 \pm 19.6 \mathrm{lb}] 47.7$ to 79.1 [105 to 174] \\
\hline
\end{tabular}

In Fig. 1 is shown a graph of $R_{i}$ (black) and $R_{e}$ (gray) averaged for all 15 subjects. Exercise began at 60 seconds and ceased at 360 seconds (each time given by the solid vertical line on the graph). Resistances stabilized while the subjects rested pre-exercise, and increased by about $15 \%$ when exercise started. There appears to be some anticipatory increase immediately before exercise began. The trend during exercise was slightly positive for inspiration and somewhat negative for expiration. From the graph, it appears that there is no easily discernible resistance difference in either $R_{i}$ or $R_{e}$ at the cessation of exercise, although there was a marked decrease in both at about 30 seconds after exercise ceased. This is about the time that the high breathing flow rates accompanying exercise diminish.

In Fig. 2 are shown data for subject 10. As can be easily noted, the variation of resistance values is more pronounced for this typical subject than it is for the averaged values appearing in Fig. 1. Most of the trends appearing in Fig. 1 are also present here: 1) there is a stable period before exercise began, 2) there was a general increase in resistances once exercising, 3) there were no discernible differences immediately upon exercise cessation, and 4) there was a general decrease about 30 seconds after exercise stopped. There was no appearance of an anticipatory increase before exercise and the general trend for both $R_{i}$ and $R_{e}$ during exercise was slightly downward. 
Averages of Inhalation and Exhalation Respiratory Resistances

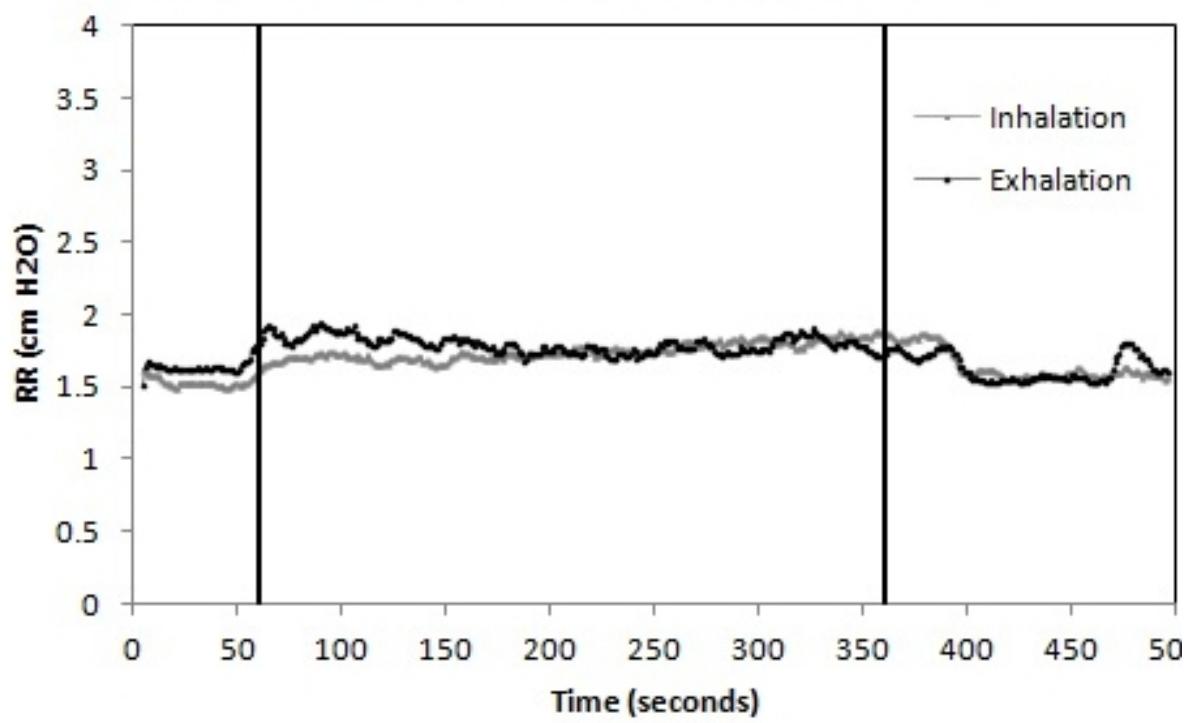

Fig. 1. Inspiratory (black) and expiratory (gray) resistance values averaged over all subject responses. Exercise began at 60 seconds and ended at 360 seconds

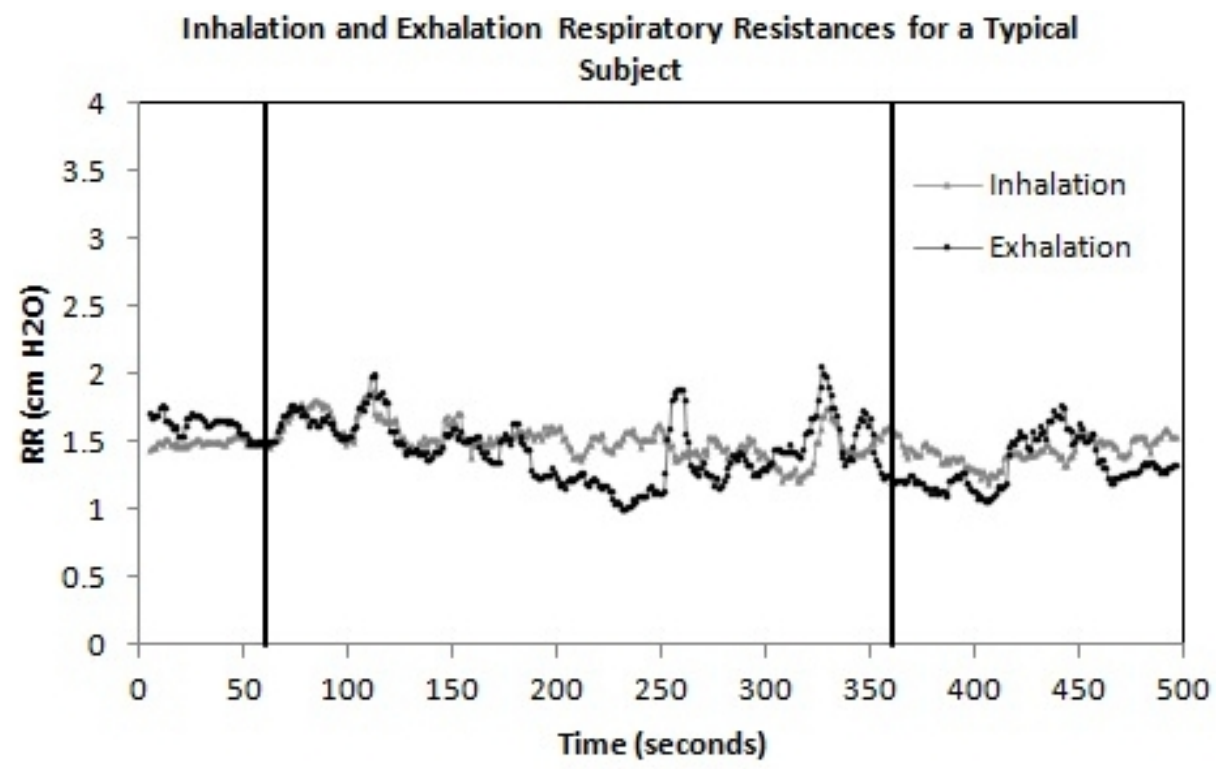

Fig. 2. Inspiratory and expiratory resistance values for subject 10 , showing resistance values before, during, and after exercise

Fig. 3 shows the flow rates averaged over all subjects during the test. Steady state was only reached toward the end of the exercise period. 


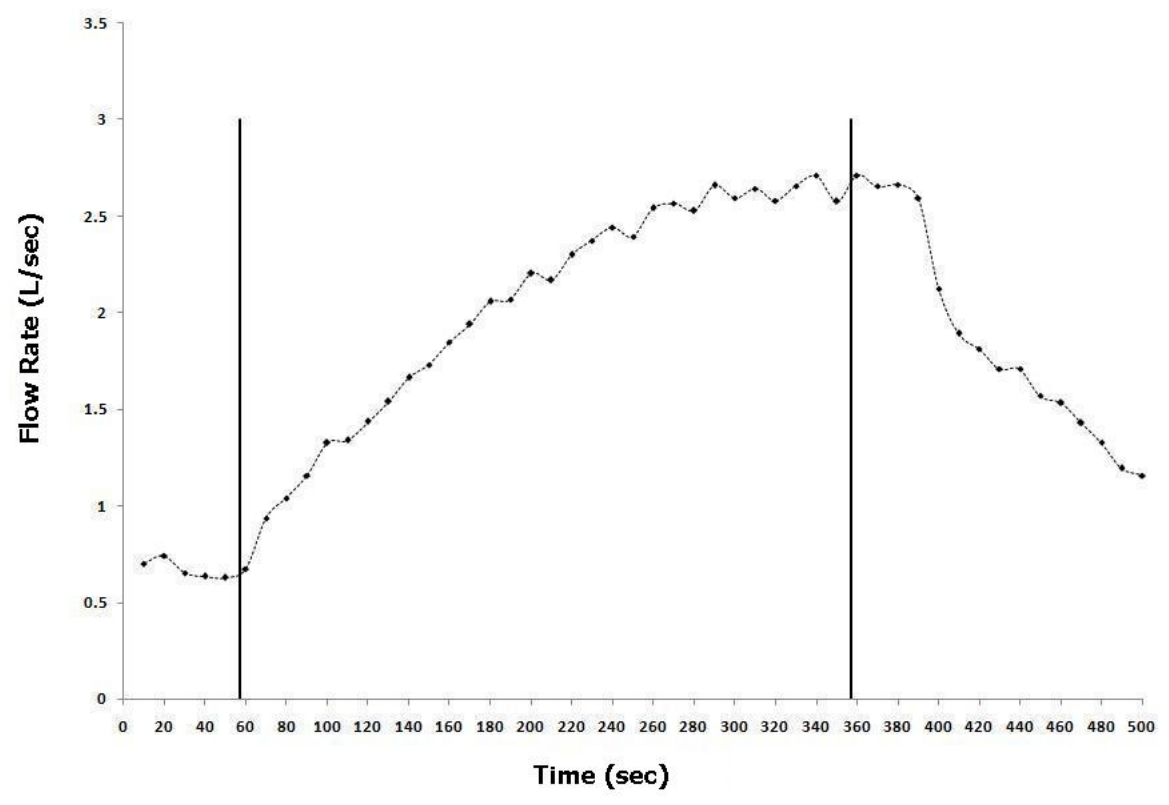

Fig. 3. Ten second averages of flow rates for all subjects

In Table 2 is a compilation of resistance differences to standard deviation ratios for each subject. No t-test run on individual subject data achieved statistical significance at the $P<.05$ level; thus individual subject resistances immediately before and after exercise ceased can be considered the same. Although no statistical test was applied to the local ratios, they are apparently of the same order of magnitude as are the statistically nonsignificant 30 second and 60 second ratios. All resistance differences are of the order of one standard deviation or less, so resistances before and after exercise ceases will likely be within a small percentage of each other for any given person. There was no apparent trend in these ratios as more samples were averaged. Therefore, averaging resistance readings over 30 or 60 seconds appears to offer no general increase in consistency before and after exercise ceases.

The calculated $t$ values for the entire cohort of subjects for local, 30 second averaging, and 60 second averaging are given at the bottom of Table 2. Shown are the unpaired and paired t-test values. All but one of these values are highly nonsignificant, so it can be expected that resistances for the entire cohort of subjects would show no significant differences before and after exercise ceases. The only value to achieve statistical significance was the inhalation resistance averaged over 60 seconds. This is reasonable when looking at Fig. 1, because average inhalation resistance appears to decrease significantly within 60 seconds after exercise ceases.

Based on the appearance of the data in Fig. 1, it appeared as if there was a significant shift in $R_{i}$ and $R_{e}$ as exercise began and also as exercise ceased. Paired observation statistical tests were performed for measurements on either side of the start of exercise and on either side of the end of exercise. For the first test, average $R_{i}$ and $R_{e}$ were formed for data appearing from 30 to 60 seconds and from 90 to 120 seconds. These values were compared as paired means with the intent to show whether the resistance increase at the beginning of exercise was statistically significant. The second test compared average $R_{i}$ and $R_{e}$ data from 330 to 360 seconds with corresponding data from 400 to 430 seconds. The intent of this test 
was to determine if the fall in resistances a short time after the end of exercise was statistically significant. Neither test achieved statistical significance. The variances of individual differences were very large, indicating that there was much variation from subject to subject. Some of this variance was caused by differences in response times; some subjects demonstrated the same trends, but the times for increases or decreases varied.

Table 2. Comparisons of sample differences to sample standard deviations at the end of exercise

\begin{tabular}{lllllll}
\hline Subject & Local ratio & \multicolumn{3}{l}{$\mathbf{3 0}$ sec ratio } & 60 sec ratio \\
\hline & Inh & Exh & Inh & Exh & Inh & Exh \\
\hline 1 & 1.035025 & -0.20051 & 0.107149 & -1.01713 & 0.898039 & 0.49386 \\
2 & 0.00697 & 0.319235 & -0.92187 & -0.85342 & 0.537749 & -0.89234 \\
3 & 0.371235 & -0.35245 & 0.965354 & 1.049834 & 0.898123 & 1.032399 \\
4 & -0.10916 & -0.20051 & -0.5316 & -1.01713 & 0.414964 & 0.49386 \\
5 & 0.153987 & 0.248836 & -1.229165 & -0.21692 & 0.308876 & 0.627609 \\
6 & -0.087439 & 0.542369 & 0.669109 & 1.298823 & 1.17987 & 1.397005 \\
7 & 0.015388 & -0.03211 & 0.393402 & 0.180411 & 1.119952 & 0.769254 \\
8 & 0.009647 & -0.08688 & 0.975555 & -0.68473 & 1.234626 & 0.469874 \\
9 & -0.16222 & -0.00401 & 1.688098 & 1.514769 & 1.074799 & 1.548484 \\
10 & 0.198229 & 0.221179 & 0.877892 & 1.502858 & 0.428099 & 1.404698 \\
11 & -0.11961 & -1.05996 & 0.735468 & 0.58048 & 0.633567 & 0.683837 \\
12 & -0.57798 & -0.200511 & -1.48109 & -1.01713 & 0.370474 & 0.49386 \\
13 & 0.132934 & 0.037933 & -0.2054 & 0.536175 & 0.502232 & 1.196191 \\
14 & -0.22245 & -0.51396 & -0.33075 & 0.184346 & 0.811614 & 1.099917 \\
15 & -0.09744 & -0.13101 & 0.002292 & -0.04607 & 0.475427 & 0.841602 \\
Averages & & & & & & \\
(t value) & -0.07143 & -0.05927 & 0.675099 & 1.073905 & 2.306372 & 1.375362 \\
(paired & -0.07143 & -0.05927 & 0.071429 & 0.071429 & 0.159487 & 0.071429 \\
t value) & & & & & & \\
\hline
\end{tabular}

Whenever statistical significance is obscured by large sample variances, nonparametric tests can sometimes be used instead. One such test is the Wilcoxon Signed Rank Test [14]. Using this test, $R_{i}$ and $R_{e}$ differences, both at the start of exercise and after exercise, were found to be statistically significant at the $P<.01$ level.

This study was undertaken mainly to provide evidence to support a procedure that would make the detection and substantiation of EIPVFM as measured with laryngoscope and APD able to be compared. Both require measurements to be taken at the mouth, and each can interfere with the other If not impossible, at least it would be extremely difficult to make both measurements simultaneously. What these results have shown is that APD measurements taken immediately before exercise stops can be compared to laryngoscopic measurements immediately after. Resistance differences for the entire population of subjects tested in this study differ by so little as to be statistically highly nonsignificant. The same is true for individual subjects. Any differences that appear are going to be small and not going to influence conclusions about laryngoscopy and APD comparisons. This applies to both inspiratory and expiratory resistances.

However, although $R_{i}$ and $R_{e}$ do not differ significantly immediately at the cessation of exercise, resistances measured a half minute or more after exercise either begins or ends 
do change significantly from their former levels. Thus, resistances increase after exercise begins and decrease after exercise ceases, as shown in Fig. 1.

For many years it was believed that airway resistance did not change during exercise because any tendency to decrease would be offset by the effect of increased breathing flow rate [15]. However, de Bisschop et al. [16] measured average respiratory resistance during exercise with an IOS system and found that resistance immediately upon the cessation of exercise was higher than at rest, but lower than what would have been expected with the higher breathing flow rate. They attributed this to reflex bronchodilation during exercise. The transient resistance they measured had disappeared by the time a second resistance measurement was made 90 seconds after exercise stopped. Results from the present study show that both inhalation and exhalation resistances decrease from their high exercise values within 45 seconds of exercise cessation.

Silverman et al. [17] used an APD to measure both inhalation and exhalation resistances at the end of exercise for subjects on a treadmill. They selected resistance values for which the breathing flow rates were within a close range to resting flow rates. Thus accounting for the effects of flow rate on resistances, they demonstrated that both inhalation resistance and exhalation resistance decreased from resting values immediately following exercise. Inhalation resistance changed more than exhalation resistance. Resistances quickly recovered to their pre-exercise values. There were no statistically significant differences between pre- and post-exercise values by 50 seconds after exercise. They were not able to measure resistances changes at the start of exercise.

The results in [17] are consistent with those of [16] and those of the present study. Resistances in Figs. 1 and 2 were not corrected for flow rate differences. These are the resistances that would be measured without selection based on flow rate, as given in Fig. 3 . de Bisschop et al. [16] implied that, if they could have corrected for increased exercise hyperpnea, the average respiratory resistances they would have measured would have been lower than pre-exercise values. The conclusion that can be reached is that resistance increases during exercise and decreases after, but this increase is the result of two opposing trends: the augmentation due to flow rate and the diminished resistance due to (probably) bronchodilation.

Normally, one would expect exhalation resistance to be slightly higher than inhalation resistance, at least at rest. This is because of the slight narrowing of the airways during exhalation compared to inhalation. Inhalation resistance is clearly shown to be somewhat larger than exhalation resistance in Figs. 1 and 2. This difference is not maintained throughout the exercise period.

To be clear about this study and its results, this study does not present a new replacement method for the diagnosis of exercise-induced paradoxical vocal fold motion (EIPVFM). If such a method comes about, it must be the result of careful and deliberate study. The APD could possibly become an important part of a new diagnostic method, but only after many careful steps. This is only the first of those steps. There is a problem that we have seen in applying laryngoscopic imaging to exercising patients, and an alternative may be more appropriate if it can be established that its indications are valid. The APD provides a pulmonary function measurement capability that has not previously been available to practitioners: the ability to easily measure respiratory resistance directly and separated into inhalation and exhalation phases. Spirometry does not do this and neither does forced oscillation. But, if it is to be used during exercise, then the APD must be validated for such 
use. If APD results are to be compared to those from the laryngoscope, then we must be sure that respiratory resistances do not change during the transition time from one measurement to the other. The results of this study confirm that respiratory resistances in normal subjects do not change immediately after exercise ceases. That is what this study has shown, and that is all this study has shown. Anything further must be tested in additional studies.

Clinical Significance: Measurements of respiratory resistance and vocal cord images during or immediately following exercise, which both require exclusive access to the mouth, cannot be made simultaneously, but this study demonstrates that both measurements can be made sequentially without loss of accuracy, as long as the two measurements are made within 30 seconds before and after the cessation of exercise. Measurements made with the APD, or other respiratory resistance measuring device, just before exercise ceases can be directly compared with laryngoscope images made soon after exercise cessation.

\section{CONCLUSION}

Both inspiratory and expiratory resistances increase at the start of exercise and decrease soon after exercise ceases. There is no statistically significant difference in either of these two resistance measurements immediately before and after exercise cessation.

\section{CONSENT}

All authors declare that 'written informed consent was obtained from the patient (or other approved parties) for publication of this case report and accompanying images.

\section{ETHICAL APPROVAL}

All authors hereby declare that all experiments have been examined and approved by the appropriate ethics committee and have therefore been performed in accordance with the ethical standards laid down in the 1964 Declaration of Helsinki.

\section{ACKNOWLEDGEMENTS}

The support of the NIH Grant R43HD062066 is greatly appreciated.

\section{COMPETING INTERESTS}

The authors have no competing interests.

\section{REFERENCES}

1. Andrianopoulos MV, Gallivan GJ, Gallivan KH. PVCM, PVCD, EPL, and irritable larynx syndrome: what are we talking about and how do we treat it? J Voice. 2000;14(4):60718.

2. Hicks M, Brugman SM, Katial R. Vocal cord dysfunction/paradoxical vocal fold motion. Primary Care Clin. Office Pract. 2008;35:81-103.

3. Gallivan GJ, Hoffman L, Gallivan KH. Episodic paroxysmal larygospasm: voice and pulmonary assessment and management. J Voice. 1996;10(1):93-105. 
4. Powell DM, Karanfilov $\mathrm{BI}$, Beechler KB, Treole K, Trudeau MD, Forrest LA. Paradoxical vocal cord dysfunction in juveniles. Arch Otolaryngol Head Neck Surg. 2000;126:29-34.

5. Balkissoon R. Vocal cord dysfunction, gastroesophageal reflux disease, and nonallergic rhinitis. Clin Allergy Immunol. 2007:19:411-26.

6. Newman KB, Mason UG, Schmaling KB. Clinical features of vocal cord dysfunction. Am J Respir Crit Care Med. 1995;152(4 Pt 1):1382-86.

7. Koufman JA, Block C. Differential diagnosis of paradoxical vocal fold movement. Am J Speech Lang Pathol. 2008;17(4):327-34.

8. Vlahakis NE, Patel AM, Maragos NE, Beck KC. Diagnosis of vocal cord dysfunction. Chest. 2002;122(6):2246-49.

9. Brugman SM, Simons SM. Vocal cord dysfunction: don't mistake it for asthma. Physician Sports Med. 1998;26:63-74.

10. Lausted CG, Johnson AT. Respiratory resistance measured by an airflow perturbation device. Physiol Meas. 1999;20:21-35.

11. Johnson AT, Sahota MS. Validation of airflow perturbation device resistance measurements in excised sheep lungs. Physiol Meas. 2004;25:679-90.

12. Lopresti E, Johnson AT, Koh FC, Scott WH, Jamshidi S, Silverman NK. Testing limits to airflow perturbation device (APD) measurements. Biomed Engr Online. 2008;7:2840, doi: 10.1186/1475-925X-7-28.

13. Johnson AT, Jones SC, Pan JJ, Vossoughi J. Variation of respiratory resistance suggests optimization of airway caliber. IEEE Trans Biomed Engr. 2012;59:2355-61.

14. Steel RGD, Torre JH. Principles and Procedures of Statistics. New York: McGraw Hill; 1960.

15. Warren J, Jennings S. Normal human airway response to exercise. J Appl Physiol. 1984;53:1686.

16. de Bisschop C, Pichon A, Guénard H, Denjean A. Accounting for flow dependence of respiratory resistance during exercise. Respir Physiol Neurobiol. 2003;136(1):65-76.

17. Silverman NK, Johnson AT, Scott WH, Koh FC. Exercise-induced respiratory resistance changes as measured with the airflow perturbation device. Physiol Meas. 2005;26:29-38.

(C) 2013 Johnson et al.; This is an Open Access article distributed under the terms of the Creative Commons Attribution License (http://creativecommons.org/licenses/by/3.0), which permits unrestricted use, distribution, and reproduction in any medium, provided the original work is properly cited.

Peer-review history:

The peer review history for this paper can be accessed here: http://www.sciencedomain.org/review-history.php?iid=205\&id=12\&aid=1183 